\title{
Fractional Quasiexcitons in Photoluminescence of Quantum Hall Liquids
}

\author{
A. WóJs ${ }^{a, b}$, A. GŁAdysiewicz ${ }^{a}$ And J.J. Quinn ${ }^{b}$ \\ ${ }^{a}$ Wrocław University of Technology \\ Wybrzeże Wyspiańskiego 27, 50-370 Wrocław, Poland \\ ${ }^{b}$ University of Tennessee, Knoxville, Tennessee 37996, USA
}

\begin{abstract}
Realistic calculations of photoluminescence spectra for a $20 \mathrm{~nm}$ quantum well at a filling factor $\nu=1 / 3$ are presented. The new states formed from charged excitons (trions) by correlation with the surrounding electrons are identified. These "quasiexcitons" differ from usual excitons and trions by having fractionally charged constituents. Their binding energies and emission intensities depend on the involved trion, leading to discontinuity in photoluminescence.
\end{abstract}

PACS numbers: 71.35.Pq, 71.35.Ji, 73.43.-f, 71.10.Pm

\section{Introduction}

The properties of a two-dimensional electron gas (2DEG) have been investigated for three decades. Photoluminescence (PL) has been used as a probe of the fractional quantum Hall effect (FQHE), and it revealed intriguing anomalies [1-4] coincident with the FQHE at filling factors $\nu=1 / 3,2 / 3$, or $2 / 5$. At low concentrations $n, \mathrm{PL}$ is dominated by emission from excitons and trions $\left(\mathrm{X}^{\prime} \mathrm{s}\right.$ and $\mathrm{X}^{-}$' $\left.\mathrm{s}\right)$. At higher $n$, interactions in the 2DEG produce Laughlin correlations among electrons, leading to the emergence of "composite fermions" (CFs) and to the FQHE. In order to perceive the optical response of incompressible Laughlin liquid, coupling of trions to the correlated 2DEG must be understood.

\section{Model}

In realistic quantum well structures, doping with donors results in an electric field, causing separation of electron and hole layers (of up to $10 \mathrm{~nm}$ in wells of width 
$w=20 \mathrm{~nm})$. In order to correctly estimate the overall impact of the electric field on the structure, first we calculated the trion binding energies for a $w=20 \mathrm{~nm}$ AlGaAs/GaAs well [5]. The energies were obtained by exact diagonalization of the $2 \mathrm{e}+\mathrm{h}$ system in the presence of magnetic field $B$ perpendicular to the well.

In order to preserve the $2 \mathrm{D}$ translational symmetry of an infinite quantum well in a finite-size calculation, we use Haldane spherical geometry [6]. Carriers are confined on a sphere of radius $R$, with magnetic monopole $2 Q<25$ (in the units of elementary magnetic flux $\phi_{0}=h c / e$ ) placed in the center. The magnetic length is $\lambda=\operatorname{sqrt}(\hbar c / e B)$, yielding $Q \lambda^{2}=R^{2}$. The extrapolation of the results to the plane is made by taking the limit $Q \rightarrow \infty$.

The single-particle states are the angular momentum $(l, m)$ eigenstates called monopole harmonics [7]. The energies fall into the $(2 l+1)$-fold degenerate angular momentum shells separated by the cyclotron energy $\hbar \omega_{\mathrm{c}}$. The quasi-2D interaction matrix elements were integrated by taking the actual e and h lowest subband wave functions in the $z$ direction. The densities $\rho_{\mathrm{e}}(z)$ and $\rho_{\mathrm{h}}(z)$ were obtained self-consistently for different electron concentrations $n$ and well widths $w$. The sample results are shown in Fig. 1.

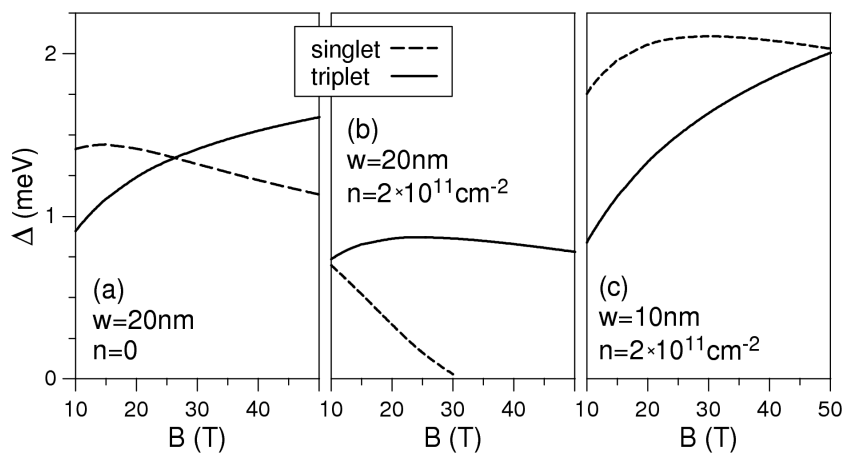

Fig. 1. Trion binding energies $\Delta$ as a function of magnetic field $B$ in an empty $w=20 \mathrm{~nm}$ well (a); $w=20 \mathrm{~nm}$ (b) and $10 \mathrm{~nm}$ wells (c) doped to $n=2 \times 10^{11} \mathrm{~cm}^{-2}$.

Notwithstanding large separation between e and $\mathrm{h}$ layers in a wider well, the $\mathrm{X}^{-}$remains bound, although its binding energy decreases significantly (Fig. 1b) compared with the situation without electric field (Fig. 1a). This trion spectrum is very similar to the spectra of ideal systems, in which the "hidden symmetry" [8] is preserved, causing the triplet to be the only bound state, and resulting in the existence of multiplicative states [9]. For narrower structures, however, the hidden symmetry is broken, resulting in imbalanced $\mathrm{e}-\mathrm{e}$ and $\mathrm{e}-\mathrm{h}$ interactions and hence in a stronger binding of the singlet state (which then becomes the ground state, see Fig. 1c). The difference in the ground state in $w=10$ and $20 \mathrm{~nm}$ structures have vast consequences on the correlations with $2 \mathrm{DEG}$ and hence on quasiexcitons emerging from the Laughlin liquid. 


\section{Quasiexcitons}

A trion $\mathrm{X}^{-}$submerged in a Laughlin liquid interacts with surrounding electrons through Haldane $\mathrm{e}-\mathrm{X}^{-}$pseudopotential [10], which produces Laughlin correlations between electrons and trions in the lowest Landau level (LL). This can be properly described in the CF framework [11] by attaching $2 p$ flux quanta to each electron or trion. All electrons converted to $\mathrm{CF}_{\mathrm{e}} \mathrm{s}$ fill the lowest LL in effective magnetic field $B^{*}=B /(2 p+1)$ at the Laughlin filling $\nu_{\mathrm{L}}=(2 p+1)^{-1}$. If $\nu>\nu_{\mathrm{L}}$, Laughlin quasiparticles will occur in the form of quasielectrons (QEs) in the excited CF-LL, each carrying effective charge $-e /(2 p+1)$. Similarly, at $\nu<\nu_{\mathrm{L}}$, the quasiholes $(\mathrm{QHs})$ will emerge from 2DEG, in a form of vacancies in the lowest CF-LL, each carrying charge $+e /(2 p+1)$. In the same way the $\mathrm{X}^{-}$in a Laughlin liquid is converted into a $\mathrm{CF}_{\mathrm{X}^{-}}$carrying fractional charge $-e /(2 p+1)$.

The $\mathrm{CF}_{\mathrm{X}^{-}}$is a many-body excitation, formed from a three-body $\mathrm{X}^{-}$by unique (Laughlin) correlation of the $\mathrm{X}^{-}$with the surrounding electrons. Its "effective" (or residual) charge $-\varepsilon$ is one (fractional) quantum of charge in a Laughlin liquid. It results from fractional (only partial) screening of a charge defect (here, $\mathrm{X}^{-}$) by the $2 \mathrm{DEG}$ in a high magnetic field. The $\mathrm{CF}_{\mathrm{X}^{-}}$will be called a negative "quasiexciton" $\mathcal{X}^{-}$.

To search for this state in an interacting $\mathrm{e}-\mathrm{h}$ liquid, we have diagonalized several different $\mathrm{Ne}+\mathrm{h}$ systems at different values of $2 Q$ (corresponding to a different number of QEs or QHs present in the Laughlin $\nu=1 / 3$ liquid) and for parameters of various realistic quantum wells (different well widths $w$ and concentrations $n$ ). Figure 2 presents the excitation energy spectra for $N=9$, with the PL oscillator strengths $\tau^{-1}$ additionally marked for each state by the radius of the open circle. Using the $\mathrm{CF}$ picture generalized for a two-component $\left(\mathrm{e}-\mathrm{X}^{-}\right)$ liquid [11], we anticipate the $\mathcal{X}^{-}$ground state with $L=2$ when $2 Q=20$. Indeed, this state is identified in Fig. 2.

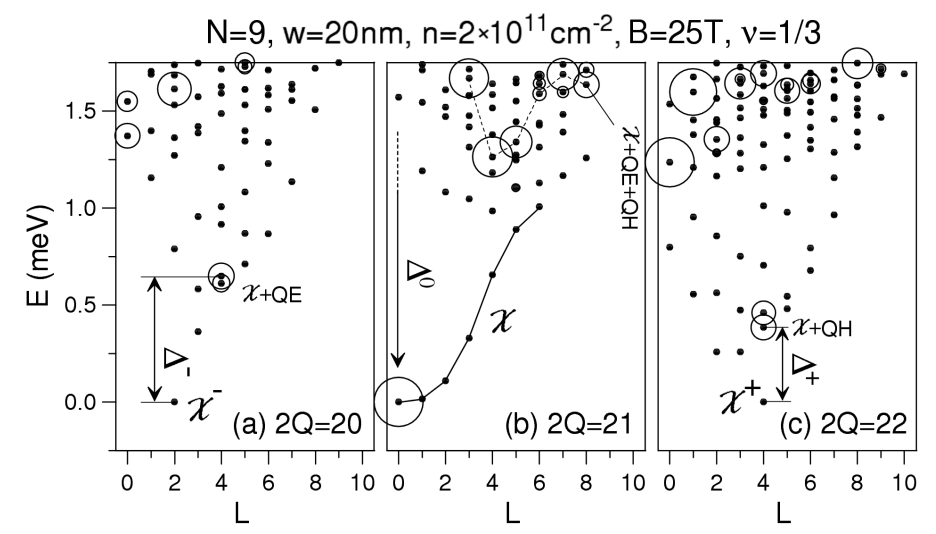

Fig. 2. Energy $E$ of the $9 \mathrm{e}+\mathrm{h}$ system as a function of total angular momentum $L$ in Laughlin $\nu=1 / 3$ state, calculated on Haldane sphere at $20 \leq 2 Q \leq 22$. 
At $2 Q=21$ there is one $\mathrm{QH}$ in addition to the $\mathcal{X}^{-}$, and the two attract each other forming a bound, neutral (quasi)excitonic state called $\mathcal{X}$. Dispersion of this state, extracted from Fig. $2 \mathrm{~b}$ is plotted in Fig. 3. At $k=0$, the $\mathcal{X}$ is approximately a multiplicative state (with a $k=0$ exciton decoupled from the remaining $N-1$ electrons). However, at $k>0$ it builds a dipole moment $d \propto k$ by splitting into fractional charge quanta of the $\mathcal{X}^{-}$and $\mathrm{QH}$ (rather than into e and h, as it is for an isolated exciton). A smaller charge of the constituents is responsible for a much shallower dispersion of the $\mathcal{X}$ compared to the "bare" $\mathrm{X}$ ). The state containing an $\mathcal{X}$ and an additional QE can be identified in Fig. 2a by its large $\tau^{-1}$. Comparison of the energies of $\mathcal{X}+\mathrm{QE}$ and $\mathcal{X}^{-}$gives the $\mathcal{X}^{-}$binding energy, $\Delta^{-}$.

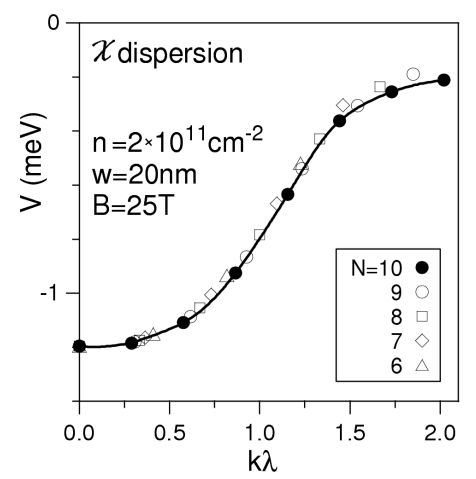

Fig. 3. Quasiexciton dispersion $\left(\mathcal{X}^{-}-\mathrm{QH}\right.$ interaction energy $V$ as a function of wave vector $k$ ) calculated from Fig. $2 \mathrm{~b}$ for the Laughlin $\nu=1 / 3$ liquid.

In Fig. 2c there are two QHs besides the $\mathcal{X}^{-}$(or, alternatively, one $\mathrm{QH}$ in addition to the $\mathcal{X})$. The ground state is a positive QX called $\mathcal{X}^{+}$at $L=4$. The identification of the nearly multiplicative of $\mathcal{X}+\mathrm{QH}$ state with a large $\tau^{-1}$ allows one to estimate the indicated binding energy $\Delta^{+}$.

The interpretation of these finite-size results for an infinite system is the following. At $\nu<1 / 3$, the $\mathcal{X}^{-}$attracts and binds a $\mathrm{QH}$ to become a neutral $\mathcal{X}^{-}+\mathrm{QH}=\mathcal{X}$ with the binding energy $\Delta^{0}=V(0)$ of Fig. 3. The $\mathcal{X}$ may capture another $\mathrm{QH}$ to become a positively charged $\mathcal{X}+\mathrm{QH}=\mathcal{X}^{+}$, with the energy gain $\Delta_{+}$. The opposite scenario happens at $\nu>1 / 3$, i.e., in the presence of QEs. The $\mathcal{X}^{+}$attracts a $\mathrm{QE}$ and, by recombination of a $\mathrm{QE}-\mathrm{QH}$ pair becomes a $\mathcal{X}$. The $\mathcal{X}$ can also annihilate another $\mathrm{QE}$ to become a $\mathcal{X}^{-}$. Whether the $\mathcal{X}$ binds a $\mathrm{QH}$ or annihilates a $\mathrm{QE}$ to acquire charge at $\nu \neq 1 / 3$ depends on the relation between $\Delta^{0}$ and the sum of $\mathrm{QE}$ and $\mathrm{QH}$ energies, $\varepsilon_{\mathrm{QE}}+\varepsilon_{\mathrm{QH}}$, which can be different in different systems. Figure 2 shows that both charged QXs are stable in a $w=20 \mathrm{~nm}$ well doped to $n=2 \times 10^{11} \mathrm{~cm}^{-2}$. The following simple relation can generally be obtained

$$
\Delta^{-}+\Delta^{0}=\varepsilon_{\mathrm{QE}}+\varepsilon_{\mathrm{QH}} .
$$




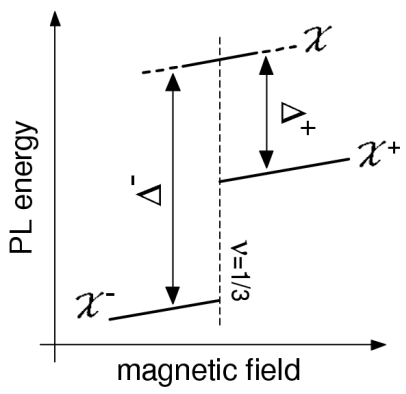

Fig. 4. Schematic of the discontinuity of the PL energy as a function of magnetic field around Laughlin $\nu=1 / 3$ filling due to emission from different quasiexcitons.

The main consequence of having different ground state for $\nu<1 / 3$ and $\nu>1 / 3$ is that, if $\Delta^{-} \neq \Delta^{+}$, a discontinuity should occur in the PL spectrum at $\nu=1 / 3$. Because the neutral $\mathcal{X}$ has a much larger oscillator strength than either $\mathcal{X}^{-}$or $\mathcal{X}^{+}$, we expect that it can also be observed in PL despite being an excited state, especially within the $\nu=1 / 3$ Hall plateau (when the QEs and QHs are localized). The predicted behavior of the emission energy near $\nu=1 / 3$ is shown schematically in Fig. 4. The values of $\Delta^{-}$and $\Delta^{+}$for our reference system (GaAs, $w=20 \mathrm{~nm}, n=2 \times 10^{11} \mathrm{~cm}^{-2}$, and $B=25 \mathrm{~T}$ ) have been estimated by extrapolation of the results similar to those in Fig. 2 but obtained for different values of $N$ and $2 Q$ to the planar limit of $\lambda / R=\sqrt{Q} \rightarrow 0$. The result is $\Delta^{-}=0.52 \mathrm{meV}$ and $\Delta^{+}=0.27 \mathrm{meV}$, in good agreement with an old (but previously unexplained) experiment of Goldberg et al. [1] and a recent observation of Byszewski et al. [2].

Let us stress that the three $\mathcal{X}$ states are in fact the same $\mathrm{X}^{-}$, only differently screened by the 2DEG. This is shown in Fig. $5 \mathrm{a}$, where the $\mathrm{e}-\mathrm{h}$ pair-distribution function $g(r)$ of the $\mathcal{X}^{+}$(the QX in which the trion is separated from the 2DEG

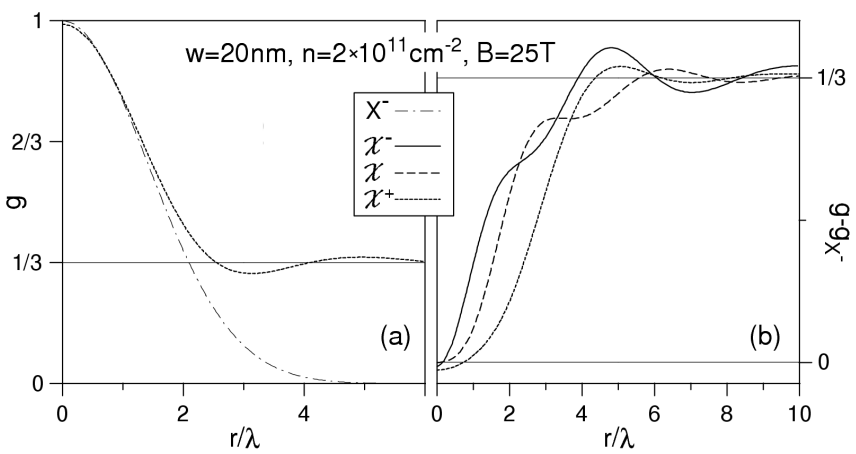

Fig. 5. (a) The e-h pair-distribution functions for quasiexciton $\mathrm{QX}^{+}$and trion $\mathrm{X}^{-}$. (b) The $\mathrm{e}-\mathrm{X}^{-}$pair-distribution functions calculated as a difference between $g(r)$ for the appropriate quasiexciton and for the trion. 
by the maximum number of two QHs) is compared to that of an isolated trion. In Fig. 5b we plotted differences between $g(r)$ for all three QXs and for the trion. This difference is the measure of the electron-trion correlations in the QX states.

\section{Acknowledgment}

We thank M. Potemski, P. Hawrylak, W. Bardyszewski, and L. Bryja for helpful discussions. Work supported by the grant DE-FG 02-97ER45657 of the U.S. Dept. of Energy and grant 2P03B02424 of the State Committee for Scientific Research (Poland).

\section{References}

[1] B.B. Goldberg, D. Heiman, A. Pinczuk, L. Pfeiffer, K. West, Phys. Rev. Lett. 65, 641 (1990).

[2] M. Byszewski, B. Chwalisz, D.K. Maude, M.L. Sadowski, M. Potemski, S. Studenikin, D.G. Austing, A.S. Sachrajda, P. Hawrylak, T. Saku, Y. Hirayama, SemiMag-16, Tallahassee (USA) 2004, unpublished.

[3] G. Yusa, H. Shtrikman, I. Bar-Joseph, Phys. Rev. Lett. 87, 216402 (2001).

[4] C. Schüller, K.-B. Broocks, P. Schröter, Ch. Heyn, D. Heitmann, M. Bichler, W. Wegscheider, T. Chakraborty, V.M. Apalkov, Phys. Rev. Lett. 91, 116403 (2003).

[5] A. Gładysiewicz, A. Wójs, J.J. Quinn, Acta Phys. Pol. A 108, 669 (2005).

[6] F.D.M. Haldane, Phys. Rev. Lett. 51, 605 (1983).

[7] T.T. Wu, Nucl. Phys. B 107, 365 (1976).

[8] I.V. Lerner, Yu.E. Lozovik, Zh. Eksp. Teor. Fiz 80, 1488 (1981).

[9] A.H. MacDonald, E.H. Rezayi, Phys. Rev. B 42, 3224 (1990).

[10] A. Wójs, I. Szlufarska, K.-S. Yi, J.J. Quinn, Phys. Rev. B 60, R11273 (1999).

[11] A. Wójs, P. Hawrylak, J.J. Quinn, Phys. Rev. B 60, 11661 (1999). 